\title{
Cloud Computing Architecture for Visual Disabilities on E-Governance
}

\author{
B. Gohin \\ Research Scholar \\ Dept. of Computer Applications \\ Dr. M.G.R. \\ Educational \& Research Institute \\ University \\ Chennai-95, Tamil Nadu, India
}

\author{
N. Rajkumar \\ Research Scholar \\ Dept. of Computer Applications \\ Dr. M.G.R. \\ Educational \& Research Institute \\ University \\ Chennai-95, Tamil Nadu, India
}

\author{
Viji Vinod \\ Phd, Professor \& Head \\ Dept. of Computer Applications \\ Dr. M.G.R. \\ Educational \& Research Institute \\ University \\ Chennai-95, Tamil Nadu, India
}

\begin{abstract}
Cloud Computing is a type of parallel and distributed system consisting of a collection of interconnected and virtualized computers that are dynamically provisioned and presented as one or more unified computing resources based on a service-level agreement. The success of the forthcoming endeavors of human civilization depends on the proper utilization of the resources which are becoming scarce day by day. It is observed that while some people have ability to use the resources, some other people with disabilities are suffering from it. This discrimination could be wiped out by a proper management and strategy adopted by the Government of different countries in the form of a properly implemented and managed E-Governance. This paper arises from our quest to understand how to design a SaaS (Software as a Service) support for more and government to explore emerging opportunities and challenges presented by "cloud computing," specifically on how it might help or hinder a more "accessible" future for people with special access needs. The existing e-Governance frameworks cannot address all categories of users. The research is intended to propose a new effective framework of E-Governance based on cloud computing concept, which would be intelligent as well as accessible by all, including people with disabilities.
\end{abstract}

\section{Keywords}

Cloud Computing, E-Governance, SaaS, Accessibility, Disabilities;

\section{INTRODUCTION}

It is beyond doubt that e-Governance can smoothen the working procedure of Government machinery by providing transparency, effective working, instant response and availability of information of Government machinery to end users, time to time. The existing e-Governance is cost effective in nature and finds itself unable to address all categories of users [2]. The success of e-Governance lies on wiping out of this discrimination by providing accessibility of different web services of e-Governance irrespective of geographical and language barriers [5]. Accessing the different web services of e-Government by using a sophisticated laptop or desktop are beyond the reach of a large number of users like visually disabled people. Hence eGovernance facilities are confined within limited inhabitants and remain unsuccessful [5]. Keeping all these in mind, we propose a new effective framework of e-Governance based on cloud computing concept. Cloud computing is computing over a cloud, where a cloud consists of clusters or grids of 1000 s of commodity machines (e.g. Linux Pcs) and software layers that is responsible for distributing application data across the machines, parallelizing and managing application executing across the machines and detecting and recovering from machine failure [8]. Here when we come to accessibility of EGovernance web Pages, we will say that accessible web design is a sign of good web design. A lot of the information on the Cloud is not accessible to people with disabilities because of poor designing of E-Governance websites [1]. While many web site managers and developers accommodate various browser constraints, most of them do not realize that they are developing sites that people with disabilities have difficulty in navigating, or in many cases. Here our proposed work is when designing E-Governance websites on cloud computing the text is large enough because low vision users usually need a screen magnifier to enlarge the text and providing audio options to notify low vision users about newer information or state changes [12]. All essential information can be accessed via text, such that blind users can use screen readers or Braille display to access the information; the information includes graphics, image maps, multimedia presentations, etc.; The cloud computing can help to make computing ubiquitous and bring it within the reach of all types of users [7]. Moreover, apart from traditional e-Governance framework, our proposed framework of e-Governance would be intelligent enough to help the end users like disabled.

The remainder of this paper is organized as follows: Section 2 cloud computing overview providing a brief review of normative literature on this research field. The purpose of this section is to explain what cloud computing really is and from what is consisted of. Thereafter, a discussion on: (2.1) Cloud Computing Service Models (2.2) deployment models of cloud computing is carried out. Section 3 Cloud Computing its Utilization For E-Governance. The purpose of this section is to highlight the benefits and limitations that derived from the migration of government services to the cloud. Section 4 Proposed Cloud Architecture for visual disabilities on eGovernance, this section explains the new effective framework for visually disabled people to use e-Governance web services on the cloud. The paper closes with conclusions and future research issues.

\section{CLOUD COMPUTING: OVERVIEW}

According to NIST, National Institute of Standards and Technology, Cloud Computing is a model for enabling convenient, on demand network access to a shared pool of configurable computing resources (e.g., Networks, servers, storage, applications, and services) that can be rapidly 
provisioned and released with minimal management effort or service provider interaction.

Cloud Computing is a general term for anything that involves delivering hosted services over the Internet. Instead of a static system architecture, Cloud Computing supports the ability to dynamically scale up and quickly scale down, offering cloud consumers high reliability, quick response times, and the flexibility to handle traffic fluctuations and demand [8]. Cloud computing also supports multi tenancy, providing systems configured in such a way that they can be pooled to be shared by many organizations or individuals [3]. Virtualization technology allows cloud vendors to convert one server into many virtual machines, thereby eliminating client-server computing with single-purpose systems [4]. This maximizes hardware capacity and allows customers to leverage economies of scale [8].

\subsection{Cloud Computing Service Models}

The service model comprises of three core options within the Cloud Computing environment.

\subsubsection{Software as a Service (SaaS)}

Sometimes referred to as "software on demand", is software that is deployed over the internet and/or is deployed to run behind a firewall on a local area network or personal computer. With SaaS, a provider licenses an application to customers either as a service on demand, through a subscription, in a "pay-as-you-go" model, or (increasingly) at no charge. This approach to application delivery is part of the utility computing model where all of the technology is in the "cloud" accessed over the Internet as a service. It is an approach whereby data is permanently stored on remote servers accessible via the Internet and cached temporarily on client devices that may include desktops, tablet computers, notebooks, handheld devices, mobile phones, etc., and is frequently called Software as a Service (SaaS) [9].

\subsubsection{Platform as a Service (PaaS)}

Perceived as Solution Stack as a service, provides an application platform, or middleware, as a service on which developers can build and deploy custom applications. PaaS offerings may include facilities for application design, application development, testing, deployment and hosting as well as application services such as team collaboration, web service integration and marshalling, database integration, security, scalability, storage, persistence, state management, application versioning, application instrumentation and developer community facilitation. These services may be provisioned as an integrated solution over the web. It facilitates the deployment of customer created applications to the cloud using provider-supported frameworks, including APIs based on Java, Python, or .Net. Although the consumers don't control the underlying cloud Infrastructure, they have control over the deployed applications and hosting environment configurations [9].

\subsubsection{Infrastructure as a Service (IaaS)}

Infrastructure as a Service is a stipulation model in which an organization outsources the equipment used to support operations, including storage, hardware, servers and networking mechanisms. When a vendor rents out infrastructure components on demand such as servers, storage components, file systems, Virtualization technologies, and network hardware the vendor is delivering an IaaS service [10]. The service provider owns the equipment and is responsible for housing, running and maintaining it. The client typically pays on a per-use basis (Amazon Elastic Compute Cloud or Amazon Simple Storage Service e.g.). A cloud service has three distinct characteristics that differentiate it from traditional hosting [13]. It is sold on demand, typically by the minute or the hour; it is elastic a user can have as much or as little of a service as they want at any given time; and the service is fully managed by the provider (the consumer needs nothing but a personal computer and Internet access). Significant innovations in Virtualization and distributed computing, as well as improved access to high-speed Internet and a weak economy, have accelerated interest in Cloud Computing [12].

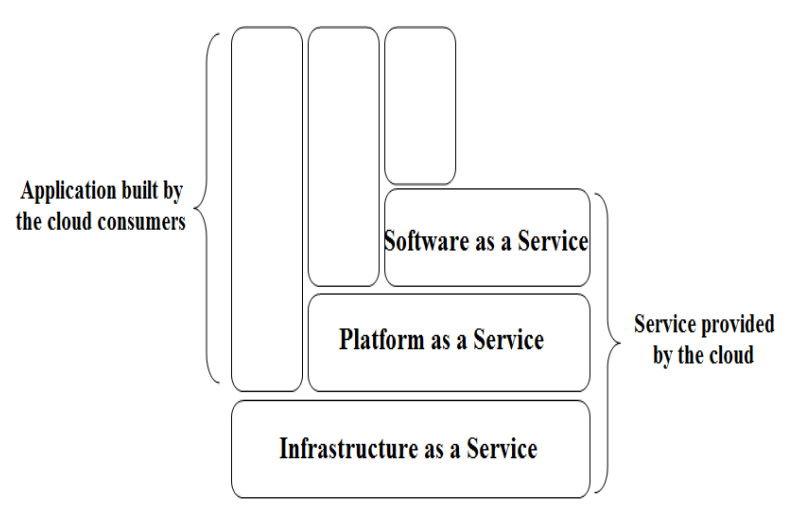

Fig 1: Cloud Computing Service Models

\subsection{Cloud Deployment Models}

In terms of implementation, there are three major types of cloud deployments; Hybrid clouds, private clouds, and public clouds.

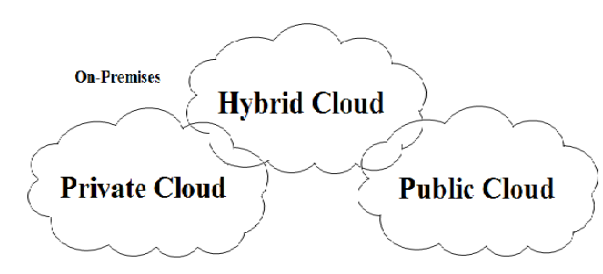

Fig 2: Cloud Deployment Models

\subsubsection{Private Clouds}

Private clouds (aka, on-premises cloud) are clouding emplacements within the organization's location, managed internally in a private cloud-based service, data and processes are administered inside the organization by an IT department within an organization, without the limitations of network bandwidth, security exposures and legal requirements that using public cloud services might involve. In addition, private cloud services present the provider and the user greater control of the cloud infrastructure, improving security and adjustability because user access and the networks used are constrained and selected. This is growing as a new architecture for the Datacenter, sometimes known as a Datacenter-in-a-box. This model gives organizations a high level of control over the use of cloud resources while bringing in the expertise needed to establish and operate the environment [12]. 


\subsubsection{Public Clouds}

Public cloud services are characterized as being "offpremises" accessible to clients from a third party service provider via the Internet. Public clouds are most often hosted away from the customer premises, and they provide a way to reduce customer risk and cost by providing a flexible, even a temporary extension of enterprise infrastructure [12]. The name public does not always signify freedom, even though it can be free or to some extent economical to use. A public cloud does not mean that a user's data is publicly available for all; public cloud vendors in fact offer an access control mechanism for their users. Public clouds provide an elastic, cost effective means to provide solutions. This type of cloud provides all the benefits of the economy of scale,

Ease of management, and ever growing elasticity.

\subsubsection{Hybrid Clouds}

In simpler terms hybrid cloud is a combination of a public and private cloud that interoperates. In this model users generally farm out no business- critical information and processing in the public cloud, while keeping business-critical services and data in their command. Hybrid Clouds are usually a combination of private clouds and public clouds, usually, managed using the same administration and monitoring consoles. A hybrid cloud also can be used to handle planned workload spikes. Sometimes called surge computing, a public cloud can be used to perform periodic tasks that can be deployed easily in a public cloud [12].

\section{CLOUD COMPUTING AND ITS UTILIZATION FOR EGOVERNANCE}

Cloud computing is a technology that uses the internet and central remote servers to maintain data and applications. Cloud computing allows consumers and businesses to use applications without installation and access their personal files on any computer with internet access. It allows for much more efficient computing by centralizing storage, memory, processing and bandwidth. Very simple example of cloud computing is Yahoo email or Gmail etc. There is no need of software or a server to use these mail facilities except an internet connection. The server and email management software is all in the cloud (internet) and is totally managed by the cloud service provider Yahoo, Google etc. The consumer gets to use the software alone and enjoy the benefits. Cloud computing provides basically three service segments: SaaS (Software as a service), PaaS (Platform as a service and IaaS (Infrastructure as a service)." Each segment serves a different purpose and offers different products for businesses and individuals around the world [1]. The most affected would be various companies and Government due to this paradigm shift .This technology can be fully utilized for various E-Governance services provided by the government at the door steps of common man [11]. In case of Companies software companies, internet service providers and hardware manufacturers would also be affected a lot if Cloud computing is being utilized properly. SaaS can be utilized for various E-Governance services (G2B, G2G, G2E, G2C). In case of PaaS database services, backup services and other middleware services can be provided effectively. IaaS allows various stakeholders to utilize the services of Hardware, Storage and Network. Cloud computing potential lies not only in the evolution of technology but also in a sourcing strategy that uses resources shared by a large number of users [6]. Cloud computing benefits may help in minimizing certain challenges of E-Governance. Cloud computing can be helpful in rolling out new projects and services. IaaS feature of cloud can be utilized for this purpose. Hence clouds can minimize the time to deploy new application instances. Corruption is a big problem in India and cloud computing can help in analyzing a huge volume of data and detecting any fraud. Corruption and red tapism can be controlled by keeping the providers of the services accountable. Cloud Virtualization technologies allow backups and restoring and hence help in disaster recovery. Cloud computing provides the facility of application migration seamlessly as compared to traditional data centers. Also various security features can be provided in the E-Governance applications with the help of Cloud computing. Cloud computing also helps in minimizing pollution. Because if we are to maintain our resources in many places it will require lots of power, air conditioning at all places but as we utilize the same infrastructure in case of a cloud computing lot of power consumption as well as the environment can be saved particularly in case of data centers. No doubt there are many benefits of cloud computing in the era of the information age. But still we hesitate in utilizing the potential of cloud computing due to various challenges of cloud computing.

\section{PROPOSED CLOUD ARCHITECTURE FOR VISUAL DISABILITIES IN E-GOVERNANCE}

In this research, we consider users with blindness, low vision and high contrast or color blindness. A blind person is one who cannot use a visual at all. These are users who read Braille displays or listen to speech output (from a screen reader) to get information from their systems. Users with low vision have a wide variety of visual capabilities. Estimates suggest that there are approximately 9-10 million people with low vision [2]. A person with low vision can be considered to be someone who can only read print that is very large, magnified, or held very close.

\subsection{Blind Users}

Designing an e-governance web site that should be relative to blind users is:

Blind users have screen reading software that can read the text contents of buttons, menus, and other control areas but screen readers cannot read the contents of an icon or image, they can only read the descriptive label or accessible name associated with them. Meaningful names should be provided for user interface objects in their code [14]. Meaningful names can allow some screen reading software to provide useful information to users with visual impairments. Rather than naming an eraser graphic "widget5", for example, the code should call it "eraser" or some other descriptive name that users will understand if spoken by a screen reader [10]. 


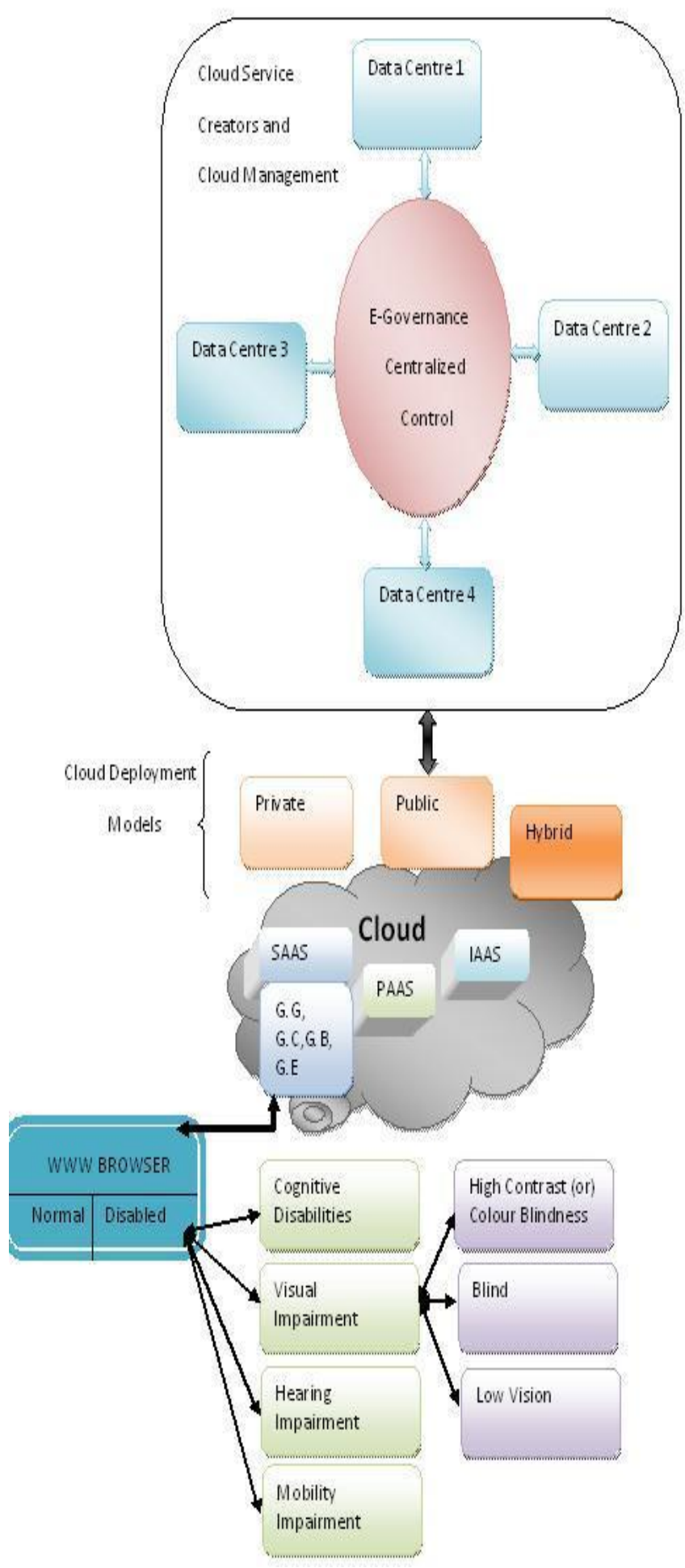

Fig 3: Cloud Architecture for Visual Disabilities

Without such descriptive information, blind or low vision users may find it difficult or impossible to interpret unlabeled, graphically labeled, or custom interface objects. Providing descriptive information may provide the only means for access in these cases. As an added selling point to developers, meaningful widget names make for code that is easier to document and debug [9].
Providing a text equivalent for every non-text element and embedded objects (e.g., via "alt", "longdesc", or in element content). This includes: images, graphical representations of text (including symbols), image map regions, animations (e.g., Animated GIFs), applets and programmatic objects, ASCII art, frames, scripts, images used as list bullets, spacers, graphical buttons, sounds (played with or without user interaction), stand-alone audio files, audio tracks of video, and video.

\subsection{Low Vision \& Color Blindness}

Our goals of accessible web design relative to low vision users are:

\section{Larger font size}

The common theme for low vision users is that it is challenging to read what is on the screen. Those who have low vision need the assistance of a hardware or software magnifier to enlarge the text beyond simple font enlargement. In E-Governance web sites use of these screen magnification hardware \& software to enlarge their view, performance and image quality are improved if larger font sizes are available prior to magnify.

Using the largest font size supported by the browser (the largest is only available when High Contrast mode is turned on).

\section{Configurable font size}

All fonts, including those in text panes, menus, labels, and information messages, can be easily configurable by users. There is no way to anticipate how large is large enough. The larger fonts can be scaled, the more likely it is that users with low vision will be able to use the software without additional magnification software or hardware.

\section{Option of audio}

A related problem for users with low vision is their limited field of view. Because of large fonts or magnify the screen through hardware or software, a smaller amount of information is visible at one time. A limited field of view means that these users easily lose context. Events in an interface outside of their field of view may go unnoticed. For these types of users we can use audio cues to corresponding texts and images on e-Governance websites. In addition, providing redundant audio cues (or the option of audio) can notify users about new information or state changes.

4. Minimize the users' memory load.

For persons using screen magnification in software services, the effective size of the computer screen can be very small. Therefore, low vision users must rely on their own memory to integrate portions of a document.

5. Use the High Contrast option in the Accessibility Options section of the Control Panel, restart the browser, and make sure the page is still readable.

6. Minimize Visual Strain, if the websites are more complex in visualizing than the low vision people can not get the correct information as they needed. So we can Use simple visual designs in e-governance web sites and Avoid patterned or complex backgrounds. So that all disabled people can get the information what they needed 
7. Resize the browser window larger and smaller to see whether there is any problem with the layout of the page because low vision users using magnification software may have a smaller effective size of the browser window.

\section{CONCLUSION}

In this paper we discussed the Cloud Computing in general and discussed how Cloud Computing can improve the accessibility of e-Governance web services based on cloud SaaS service model. The SaaS model of service provision has the potential to become an important sourcing strategy for government organizations. SaaS is a type of service model to deliver software as a service instead of owning and maintaining software locally. Disabled people may have stronger needs for or benefits from the e-Governance services than the general population, but they have a lower usage rate. The main gate to access the E-government websites ( $\mathrm{SaaS}$ ) is browsing. As browsing the existing website is designed to cater to only normal people with no disabled people. But here, neither we have proposed cloud architecture for accessing the e-Governance web services to visual \& cognitive disabilities only nor other disabilities like mobility impaired and hearing impaired. We intend to do so in our forthcoming endeavors.

\section{REFERENCES}

[1] Cloud Computing. Wikipedia, Available at http://en.wikipedia.org/wiki/Cloud_computing. .

[2] Daniel Oliveira and Eduardo Ogasawara. Article: Is Cloud Computing the Solution for Brazilian Researchers?, International Journal of Computer Applications 6 (8): 19-23, September 2010.

[3] D. Oliveira, F. Baiao, and M. Mattoso, 2010, "Towards Taxonomy for Cloud Computing from an e-Science Perspective", Cloud Computing: Principles, Systems and Applications (to be published), Heidelberg: SpringerVerlag.

[4] I. Foster, Y. Zhao, I. Raicu, and S. Lu, 2008, Cloud Computing and Grid Computing 360-Degree Compared, In: Grid Computing Environments Workshop, 2008. GCE '08, p. 10,1 .

[5] K.Mukherjee and G.Sahoo. Article: Cloud Computing: Future Framework for e-Governance. International Journal of Computer Applications 7(7):31-34, October.
[6] Liang-Jie Zhang, Qun Zhou, "CCOA: Cloud Computing Open Architecture," icws, pp.607-616, 2009 IEEE International Conference on Web Services, 2009.

[7] Liladhar R Rewatkar and Ujwal A Lanjewar. Article: Implementation of Cloud Computing on Web Application. International Journal of Computer Applications 2(8):28-32, June 2010. Published By Foundation of Computer Science.

[8] L.M. Vaquero, L. Rodero-Merino, J. Caceres, and M.Lindner, 2009, A break in the clouds: towards a cloud definition, SIGCOMM Comput. Commun. Rev., v. 39, n. 1, p. 50-55.

[9] Rajarshi Chakraborty, Srilakshmi Ramireddy, T.S. Raghu,H. Raghav Rao, - The Information Assurance Practices of Cloud Computing Vendors, IT Pro July/August 2010, In IEEE Computer Society, p. 29-37.

[10] S. Bhardwaj, L. Jain, and S. Jain, —Cloud Computing: Cloud Computing: A Study of Infrastructure as a Service (IaaS), Int ${ }^{\star} 1$ J. Eng. and Information Technology, vol. 2, no. 1,2010 , pp. 60-63.

[11] Sonal Anand, Sarvesh Gupta, Shweta Fatnani, Varsha Sharma and Deepti Jain. Article: Semantic Cloud for Mobile Technology. International Journal of Computer Applications 8(12):1-4, October 2010.

[12] Sun Microsystems White Paper, - Introduction to Cloud Computing Architecture, June 2009.

[13] The Future of Cloud Computing, Opportunities for European Cloud Computing Beyond 2010. Expert Group Report. European Commission Information Society and Media, Public Version 1.0, pp 12.

[14] VOGELS, W. A Head in the Clouds - The Power of Infrastructure as a Service. In First workshop on Cloud Computing and in Applications (CCA $=08$ ) (October 2008).

[15] W.K. Chan, Lijun Mei, and Zhenyu Zhang, "Modeling and testing of cloud applications", to appear in Proceedings of 2009 IEEE Asia-Pacific Services Computing Conference (APSCC 2009), (Singapore, December 7-11, 2009), IEEE Computer Society Press, Los Alamitos, CA, USA, 2009.

[16]Y. Zhao, I. Raicu, and I. Foster, 2008, Scientific Workflow Systems for 21st Century, New Bottle or New Wine? In: 2008 IEEE Congress on Services, p. 467-471 